\title{
Estabilização de fratura de complexo zigomático-orbitário com dois pontos de fixação: relato de caso
}

Stabilization of zygomatic-orbitary complex fracture with two point fixation: case report Estabilización de fractura de complejo zigomático-orbitario con dos puntos de fijación: reporte de caso

\author{
Mateus Barros CAVALCANTE1 \\ Caio Pimenteira UCHÔA 1 \\ Caroline Brígida Sá ROCHA1 \\ Luiz Henrique Soares TORRES ${ }^{2}$ \\ Valfrido Antônio PEREIRA FILHO² \\ Ialle Micaelle Pereira MARCELINO \\ Emanuel Dias de OLIVEIRA E SILVA4 \\ Faculdade de Odontologia de Araraquara, 14801-903, Araraquara-SP, Brasil
}

${ }^{1}$ Residente em Cirurgia e Traumatologia Bucomaxilofacial do Hospital Universitário Oswaldo Cruz, Universidade de Pernambuco (UPE), 50100-130 Recife - PE, Brasil ${ }^{2}$ Programa de Pós-graduação em Ciências Odontológicas, Departamento de Diagnóstico e Cirurgia, Universidade Estadual Paulista “Júlio de Mesquita Filho” (UNESP) ${ }^{3}$ Cirurgiã-Dentista pela Universidade Federal de Pernambuco (UFPE), 50670-901, Recife - PE, Brasil

${ }^{4}$ Departamento de Cirurgia e Traumatologia Bucomaxilofacial, Hospital Universitário Oswaldo Cruz, UPE Universidade de Pernambuco, 50100-130 Recife-PE, Brasil

\begin{abstract}
Resumo
Introdução: o zigoma articula-se com quatro ossos e é de extrema importância na força e estabilidade do terço médio da face. Por sua projeção anterior na face, torna-se um osso bastante susceptível aos traumas. Fraturas podem resultar em sequelas funcionais significantes e estéticas. Objetivo: relatar um caso clínico de fratura de zigoma, bem como debater sobre possíveis formas de fixação, suas indicações e sequencia cirúrgica. Materiais e métodos: paciente gênero masculino, 26 anos de idade e com histórico de agressão física. Ao exame clínico e de imagem, constatou-se fratura de complexo zigomático-orbitário direito, que foi tratado com osteossíntese em dois pontos de fixação. Resultados: a abordagem de fixação da fratura nas suturas zigomático-frontal e zigomático-maxilar foi satisfatória e concedeu estabilidade e projeção anteroposterior na face. Paciente segue em acompanhamento de seis meses sem apresentar sequelas ou complicações. Conclusões: o manejo das fraturas do complexo zigomático-maxilar ainda é discutido e controverso na literatura. Porém a abordagem com fixação de dois pontos se mostrou precisa, menos invasiva e com menor custo.
\end{abstract}

Descritores: Zigoma; Fixação de Fratura; Traumatologia.

\section{Abstract}

Introduction: The zygoma is articulated with four bones and is of extreme importance in the strength and stability of the middle third of the face. By its anterior projection on the face, it becomes a bone quite susceptible to trauma. Fractures may result in significant functional and aesthetic sequelae. Objective: to report a clinical case of zygoma fracture, as well as the form of assessment, its indications and surgical sequence. Materials and methods: male patient, 26 years of age and with a history of physical aggression. At the clinical and imaging examination, a right zygomatic-orbitary complex fracture was observed, which was treated with osteosynthesis at two fixation points. Results: the fracture fixation approach in the zygomatic-frontal and zygomatic-maxillary sutures was satisfactory and provided anteroposterior stability and projection on the face. In a six months follow up there are no sequelae or complications. Conclusions: the management of fractures of the zygomatic-maxillary complex is still discussed and controversial in the literature. However, the two-point fix was more accurate, less invasive and less costly.

Descriptors: Zygoma; Fracture Fixation; Traumatology.

\section{Resumen}

Introducción: el zigoma se articula con cuatro huesos y es de extrema importancia en la fuerza y estabilidad del tercio medio de la cara. Por su proyección anterior en la cara, se convierte en un hueso bastante susceptible a los traumas. Las fracturas pueden resultar en secuelas funcionales significantes y estéticas. Objetivo: relatar un caso clínico de fractura de zigoma, así como debatir sobre posibles formas de fijación, sus indicaciones y secuencia quirúrgica. Materiales y métodos: paciente género masculino, 26 años de edad y con historial de agresión física. En el examen clínico y de imagen, se constató una fractura de complejo zigomático-orbitario derecho, que fue tratado con osteosíntesis en dos puntos de fijación. Resultados: el enfoque de fijación de la fractura en las suturas zigomático-frontal y zigomáticomaxilar fue satisfactoria y concedió estabilidad y proyección anteroposterior en la cara. El paciente sigue en seguimiento de seis meses sin presentar secuelas o complicaciones. Conclusiones: el manejo de las fracturas del complejo zigomático-maxilar todavía es discutido y controvertido en la literatura. Pero el enfoque con fijación de dos puntos se mostró precisa, menos invasiva y con menor costo.

Descriptores: Zigoma; Fijación de Fractura; Traumatología.

INTRODUÇÃO

As fraturas faciais apresentam um papel importante no manejo de pacientes politraumatizados em hospitais de emergências pelo fato da região maxilofacial ocupar uma posição proeminente no corpo humano, sendo bastante vulnerável aos traumas ${ }^{1}$. O osso zigomático, devido a sua projeção anterior no esqueleto facial, é uma estrutura altamente susceptível a esse tipo de injúria, interferindo na forma e função facial ${ }^{2}$. As fraturas dos osso zigomáticos são apenas menores quando comparado às lesões aos ossos próprios do nariz ${ }^{3}$.

O Complexo Zigomático-Orbitario (CZO) possui forma de pirâmide quadrangular e se articula com quatros ossos através das suturas zigomaticofrontal, zigomaticotemporal, zigomaticomaxilar e zigomaticoesfenóide, normalmente zonas de fratura. Compõe uma área estética e funcional do esqueleto facial, serve como barreira óssea e separa estruturas anatômicas importantes com órbita, maxilares e fossa temporal ${ }^{4}$. Está relacionado com a dissipação de forças da mastigação, sobretudo pela presença dos pilares faciais, além de ser responsável pelas projeções ântero-posterior e láterolateral da face ${ }^{5}$.

$\mathrm{O}$ diagnóstico das fraturas do $\mathrm{CZO}$ costuma ser estabelecido pela história clínica da lesão, exame físico detalhado, além das tomografias computadorizadas em cortes nos planos axiais e coronais, e radiografias convencionais como as projeções de Waters, Cawdel e Hirtz ${ }^{4}$. Os sinais e 
sintomas mais prevalentes de trauma nessa região são assimetria facial por perda da projeção anterior do osso zigomático, descontinuidade óssea palpável na sutura fronto-zigomática e margem infraorbitária, dor, parestesia em asa do nariz, lábio superior e dentes anteriores superiores e epistaxe ${ }^{4}$.Alguns trabalhos relatam quadros clínicos que envolvem déficit visual e funcional, pelo envolvimento da porção do assoalho orbital ${ }^{6}$.

$\mathrm{Na}$ maioria dos casos as fraturas de CZO são tratadas após quarto ou cinco dias após a lesão, tempo necessário para a resolução do edema ${ }^{6} \mathrm{e}$ melhora dos sintomas, o que facilita, por exemplo, as manobras do acesso cirúrgico. Apesar da alta frequiência de fraturas do $\mathrm{CZO}$, ainda não há consenso entre cirurgiões Buco-Maxilo-Faciais sobre o melhor manejo dessas lesoes, portanto a abordagem cirúrgica para esse tipo de fratura ainda é desafiador ${ }^{7}$.

Quatro princípios básicos devem ser considerados ao realizar o reparo de uma fratura: exposição e redução adequadas, fixação estável e complicações mínimas. O tratamento visa restaurar e manter a configuração esquelética facial prétrauma $^{8} \mathrm{e}$ baseia-se na utilização de técnicas incruentas ou cruentas, com métodos de fixação interna estável com o uso de miniplacas e parafusos de titânio em zonas se sustentação especificas $^{9,10}$.

O presente trabalho tem o objetivo de relatar um caso clínico de paciente vítima de fratura do complexo zigomático orbitário, tratado com fixação interna funcionalmente estável em dois pontos de fixação.

\section{CASO CLÍNICO}

Paciente gênero masculino, 26 anos, compareceu ao Hospital da Restauração (Recife/PE) vítima de agressão física. Após avaliação médica inicial, constatou-se ao exame físico a presença de discreto edema e equimose peri-orbitária à direita, dor à palpação, motricidade ocular e acuidade visual preservadas, sugerindo hipótese diagnóstica de fratura em complexo zigomático orbitário. Ao exame de tomografia computadorizada de face, observou-se traços de fratura em CZO direito (Figura 1).

Após discussão com equipe, indicou-se procedimento cirúrgico para redução e fixação das fraturas. O procedimento cirúrgico foi realizado sob anestesia geral e intubação por via nasotraqueal. Foi realizado acesso em região supraciliar direita e acesso intrabucal vestibular maxilar à direita. Após a exposição e mobilização dos remanescentes ósseos fraturados com auxilio do ganho de Barros, as fraturas na região súperolateral e, após checagem da redução através da sutura zigomático-esfenoidal o pilar zigomático-maxilar direito foram reduzidas e fixadas com placa e parafusos do sistema $2.0 \mathrm{~mm}$ (Figura 2). As feridas cirúrgicas foram suturadas por

planos com fios reabsorvíveis e, em pele, com mononylon 5-0. No pós-operatório imediato, foi realizado teste da ducção forçada confirmando a liberação periorbitária muscular (Figura 2). A alta hospitalar ocorreu após $24 \mathrm{~h}$ de internamento. O paciente foi acompanhado por avaliação clínico-radiográfica ambulatorial semanalmente. Após seis meses de pósoperatório, não foi observado complicações ou sequelas (Figuras 3 e 4 ).
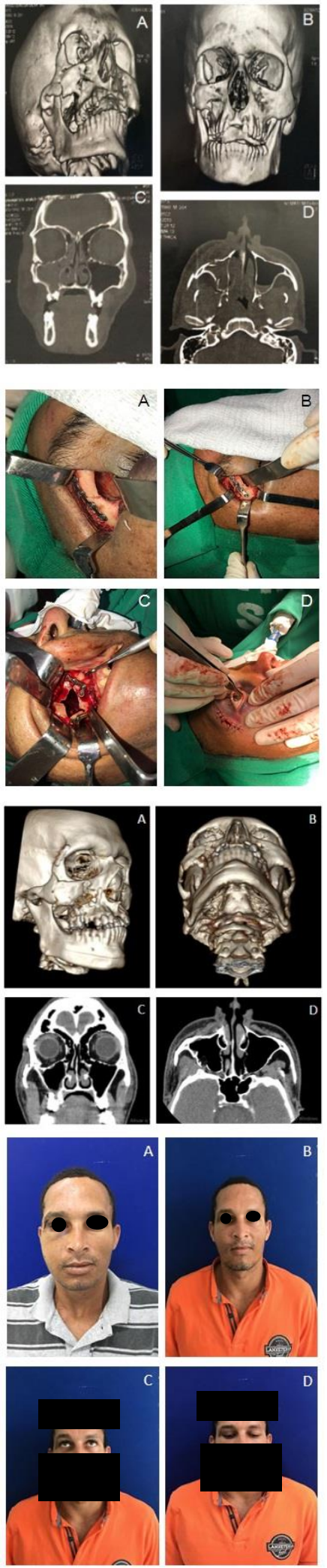

Figura 1: A. Vista obliqua de fratura de CZO em reconstrução tridimensional de tomografia computadorizada. B. Vista frontal de fratura de CZO em reconstrução tridimensional de tomografia computadorizada. C. Corte coronal de tomografia computadorizada pré-cirúrgica. D. Corte axial de tomografia computadorizada pré-cirúrgica.

Figura 2: A. Checagem transoperatória da redução da sutura esfeno-zigomática B. Osteossíntese do pilar fronto-zigomático.

C. Osteossíntese do pilar zigomático-maxilar.

D. Realização do teste de ducção forçada.

Figura 3: A. Vista obliqua de reconstrução tridimensional de tomografia computadorizada pós-cirúrgica.

B. Vista frontal de reconstrução tridimensional de tomografia computadorizada pós-cirúrgica.

C. Corte coronal de tomografia computadorizada pós-cirúrgica. D. Corte axial de tomografia computadorizada pós-cirúrgica.

Figura 4: A. Acompanhamento clínico de uma semana.

B. Simetria facial em vista frontal em acompanhamento clínico de seis meses.

C. Movimentos oculares de supra-versão preservados.

D. Movimentos oculares de infra-versão preservados. 


\section{DISCUSSÃO}

Dentre as estruturas ósseas da face, osso zigomático, devido a sua anatomia e projeção anterior no esqueleto, é comumente envolvido em lesões traumáticas ${ }^{3}$. Apesar de forte, o zigoma, apresenta zonas de fragilidade, seus quatro processos: frontal, orbital, maxilar e temporal que são frequentemente fraturados ${ }^{11}$. Dentre estas estruturas, a sutura fronto-zigomática é uma conexão fina e delicada que fratura frequentemente com as mínimas forças. Contudo a depender do mecanismo do trauma e energia dissipada, não somente esta sutura é acometida, mas sim todas as outras, incluindo até mesmo o corpo do zigoma.

Diversos trabalhos descrevem que em casos onde exista deslocamento e instabilidade óssea, a redução aberta e fixação interna têm sido usadas como o método padrão para o tratamento de fratura de $\mathrm{CZO}^{11,12}$. Dependendo da estabilidade do zigoma reduzido, as fixações de um, dois e três pontos podem ser aplicadas. A escolha do tipo de fixação utilizadas em cada caso irá depender de alguns fatores como redução anatômica do complexo zigomático e estabilidade adequada e posicionamento tripóide do zigoma $^{13}$. Embora a fixação de três pontos proporcione maior estabilidade, existem relatos de casos selecionados, que a fixação em um único ponto é suficiente para manter a estabilidade ${ }^{9,14}$

Alguns experimentos biofísicos foram realizados para descobrir a estabilidade rotacional da fratura após fixação com mini-placas. Davidson e colaboradores $^{15}$ analisaram diferentes combinações de fixação para estabilizar uma fratura de $\mathrm{CZO} \mathrm{em}$ crânios humanos e descobriram que a fixação de três pontos na sutura fronto-zigomatica, margem infraorbitária e pilar zigomaticomaxilar conferiu estabilidade máxima contra forças correspondentes de estresses fisiológicos.

Em 2011, Kim et al. ${ }^{16}$ compararam o uso de um ou dois pontos de fixação em fraturas de CZO. No primeiro grupo, as fraturas foram tratadas apenas com um ponto de fixação, o pilar zigomático-maxilar e o segundo grupo as fraturas foram fixadas em dois pontos, o pilar zigomático-maxilar e a sutura frontozigomática - assim como no neste caso. Os autores concluem que a utilização de apenas um ponto de fixação confere uma alta estabilidade cirúrgica e evita a formação de cicatrizes indesejáveis em casos selecionados.

Poucos trabalhos clínicos discutem e analisam os resultados do emprego de um, dois e três pontos de fixação em pacientes com fraturas de $\mathrm{CZO}^{8,17}$. Em 2017, Nasr et al. ${ }^{18}$ compararam a utilização de dois e três pontos de fixação em pacientes com fraturas de zigoma. Os autores relatam que a fixação de três pontos é a técnica padrão, contudo a modalidade com dois pontos é efetiva, clinicamente imperceptível e quase tão eficaz quanto à fixação de três pontos impedindo a rotação ou deslocamento ósseo, alem de ter menor custo.

Em 2014, Ellis et al. ${ }^{10}$ estudando as fraturas de CZO, suas fixações e sequencia cirúrgica, definiram um algoritmo de tratamento a ser aplicado neste tipo de trauma. Com o objetivo de minimizar o número de acessos necessários na tentativa de diminuir o potencial iatrogênico de deformidades estéticas, o autor conclui que na maioria dos casos de fraturas de complexo zigomático seu algorítimo se apliacaria e que o uso da tomografia computadorizada transoperatória (TCT) permite ao cirurgião ser menos invasivo, com maior previsibilidade e precisão. Embora neste relato, nao se tenha realizado TCT, aplicou-se o plano de tratamento proposto por Ellis et al. ${ }^{10}$ abordando apenas dois pontos: primeiro a sutura frontozigomática seguida do pilar zigomatico-maxilar. Com o intuito de minimizar a quantidade de acessos cirúrgicos diminundo tempo operatório e deformidades estéticas. O que pode ser realizado pelo fato da correta redução óssea, pela estabilidade alcançada com dois pontos de fixação e de não haver necessidade de reconstrução orbitária.

CONCLUSÃO

Ainda que o manejo das fraturas do complexo zigomático-orbitário seja um tema discutido e controverso na literatura, a utilização de dois pontos de fixação se mostrou satisfatório neste caso relatado, alcançando boa estabilidade e um adequado posicionamento tridimensional. Quando bem indicada, esta abordagem pode ser aplicada no tratamento dessas fraturas, a fim de se estabelecer a forma e função facial, além de minimizar o tempo operatório, cicatrizes desnecessárias e custo.

\section{REFERÊNCIAS}

1. Nagaprasad N, Praveen Harish G, Reddy GSG. Clinical Study of Isolated Zygomatic Fractures. Int J Contemp Med Res. 2016;3(7):1983-86.

2. Jardim ECG, Santiago Junior JF, Melo RL, Mendonça JCG, Faverani LP, Garcia Júnior IR et al. Combinação de técnicas para tratamento cirúrgico de fratura do complexo zigomáticomaxilar: relato de caso. Arch Health Invest. 2013; 2(3):33-6.

3. Sassi LM, Dissenha JL, Bezeruska C, Guebur MI, Hepp V, Radaelli RL et al. Fraturas de zigomático: revisão de 50 casos. Rev Bras Cir Cabeça Pescoço. 2009; 38:246-7.

4. Mendonça JCG, Crivelli DMB. Tratamento de fratura cominutiva do complexo zigomático orbitário com utilização de fio de aço: relato de caso. Rev Bras Cir Cabeça Pescoço. 2012;41(2):93-5.

5. Oliveira JAGP. Fratura do arco zigomático: relato de caso utilizando o acesso pré-auricular. Rev Cir Traumatol Bucomaxilofac. 2009; 9(1):47-52. 
6. Oliva MA. Acesso subciliar para fraturas do complexo zigomático-orbitári. Rev Bras Cir Cabeça Pescoço. 2013;42(2):106-8.

7. Meslemani D, Kellman RM. Zygomaticomaxillary complex fractures. Arch Facial Plast Surg. 2012; 14(1):62-6

8. Rana M, Warraich R, Tahir S, Igbal A, von See C, EcKardt AM et al. Surgical treatment of zygomatic bone fracture using two points fixation versus three point fixation- a randomised prospective clinical trial. Trials 2012; 13:36

9. Ellis E 3rd, Kittidumkerng W. Analysis of treatment for isolated zygomatic maxillary complex fractures. J Oral Maxillofac Surg. 1996; 54(4):386-400.

10.Ellis E 3rd, Daniel Perez. An algorithm for the treatment of isolated zygomatico-orbital fractures. J Oral Maxillofac Surg. 2014;72(10):1975-83.

11. Tripathi N, Goyal M, Mishra B, Dhasmana S. Zygomatic complex fracture: A comparative evaluation of stability using titanium and bioresorbable plates as one point fixation. Nat $\mathbf{J}$ Maxillofac Surg. 2013;4(2):181-87.

12. Yonehara $\mathrm{Y}$, Hirabayashi S, Tachi M, Ishii $\mathrm{H}$. Treatment of zygomatic fractures without inferior orbital rim fixation. J Craniofac Surg. 2005;16(3):481-85.

13.Eski M, Sahin I, Deveci M, Turegun M, Isik S Sengezer M. A retrospective analysis of 101 zygomatico-orbital fractures. J Craniofac Surg. 2006;17(6):1059-64.

14. Chrcanovic BR, Cavalcanti YS, Reher P. Temporal miniplates in the frontozygomatic areaan anatomical study. J Oral Maxillofac Surg. 2009;13(4):201-6.

15. Davidson J, Nickerson D, Nickerson B. Zygomatic fractures: comparison of methods of internal fixation. Plast Reconstr Surg. 1990;86(1):25-32.

16. Kim ST, Godh, Jung JH. Comparison of one point fixation with two point fixation in treating tripod fractures of zygoma, J Oral Maxillofac Surg. 2011; 69(11):2848-52

17. Kovács AF, Ghahremani M. Minimization of zygomatic complexfracture treatment. Int $\mathrm{J}$ Oral Maxillofac Surg. 2001; 30(5):380-83.

18. Nasr WF, ElSheikh E, El-Anwar MW, Sweed AH, Bessar A, Ezzeldin N. Two-versus threepoint internal fixation of displaced zygomaticomaxillary complex fractures. Craniomaxillofac Trauma Reconstr. 2018;11(4):256-64.

\section{CONFLITO DE INTERESSES}

Os autores declaram não haver conflitos de interesse.

\section{AUTOR PARA CORRESPONDENCIA}

\section{Caio Pimenteira Uchôa}

caiopuch@gmail.com
Submetido em 29/12/2018

Aceito em 06/02/2019 